\title{
RADICAL-BASED TUNING THE SURFACE FUNCTIONALITY OF MXENE
}

\author{
1,2Anastasiia OLSHTREM, ${ }^{3}$ Sergii CHERTOPALOV, 1,2Olga GUSELNIKOVA, ${ }^{1}$ Vaclav SVORCÍK, \\ ${ }_{1,2}$ Oleksiy LYUTAKOV
}

${ }^{1}$ VŠCHT - University of Chemistry and Technology, Prague, Czech Republic, EU, Iyutakoo@vscht.cz

${ }^{2}$ Tomsk Polytechnic University, Research School of Chemistry and Applied Biomedical Sciences, Tomsk, Russian Federation

${ }^{3}$ Institute of Physics of the Czech Academy of Sciences, Prague, Czech Republic, EU

https://doi.org/10.37904/nanocon.2020.3770

\begin{abstract}
The family of MAX phases and their derivative MXenes are continuously growing in terms of both crystalline and composition varieties. MXenes are a new family of two-dimensional (2D) transition metal carbides, carbonitrides and nitrides, with a general formula $M n+1 A X n$, where $n=1-3, M$ denotes a transition metal, $A$ is an element such as aluminum or silicon, and $X$ is either carbon or nitrogen. Considering the various elemental composition possibilities, surface functional tunability, various magnetic orders, and large spin-orbit coupling, MXene can truly be considered as multifunctional materials that can be used to realize highly correlated phenomena. However, a change in surface chemical groups can significantly alter the properties and functionality of MXene flakes and may even damage the flakes. In this paper, we propose the possibility of using soft, chemical transformation to tune the MXene surface chemistry and termination. We used fluorinated and brominated substituents for MXenes grafting and subsequent analysis of the surface composition of the MXene flakes indicated a decrease in oxygen and a simultaneous increase in fluorine and bromine surface concentrations. The ability to graft organic groups with various substituents to the surface of the flakes opens up new possibilities for their application. The presence of fluorinated groups on the surface makes it hydrophobic, which allows the creation of water-repellent flakes, and prevents rapid oxidation by atmospheric oxygen and related formation of titanium oxide on the surface.
\end{abstract}

Keywords: Chemical transformation, MXene, Surface grafting, Tuned functionalization

\section{INTRODUCTION}

Creation and study of two-dimensional (2D) materials is a young and very promising area of modern materials science [1-4]. These materials are very thin (often less than one $\mathrm{nm}$ ), so they can be used to create layered heterostructures that are used in modern electronics, from transistors to sensors, solar cells and LEDs [5-10]. The most famous two-dimensional material is graphene, its discovery in 2010 was awarded the Nobel Prize in physics [11]. Graphene possesses high values of electrical and thermal conductivity and is sufficiently strong in tensile strength [12-15]. At the same time, it forms folds and is unstable as a separate free-hanging film $[16,17]$. Discovered in 2011, the MXene have surpassed graphene in both electrical characteristics and manufacturability [18,19]. MXene exhibits conductivity superior to graphene coating. In addition, MXene combines many characteristics, including high shielding efficiency, low density, low thickness, high flexibility and ease of processing [20-22]. So, it is a great candidate for numerous applications, such as transparent conductive coatings, protective layers, and electrochemically-active supports [23, 24].

It has been proven that the surface chemistry of Mxene affects its conductive properties, which can be controlled and even rearranged. However, methods of modifying the surface chemistry of MXene flakes often damage the MXene structure. 
In this contribution, we propose a new approach for modifying a new and interesting class of 2D materials Mxenes with formula Ti3AIC2. Our approach is based on the surface modification under attack by higly reactive radicals.

\section{EXPERIMENTAL}

\subsection{Materials}

3,5-Bis(trifluoromethyl)phenyl-boronic acid ( $\geq 95 \%)$, 1-iodo-3,5-bis(trifluoromethyl)benzene (98\%), boron trifluoride diethyl etherate (99\%), 3-Chloroperbenzoic acid ( $\leq 77 \%)$, methanol and were purchased from SigmaAldrich. Powder of titanium (99\%), aluminum (99.5\%) and graphite (99\%) were purchased from Alfa Aesar. All reagents were used without further purification. Throughout the all experiments deionized water was used. Ti3AIC2 MAX phase and Mxene flakes were prepared according to $[25,26,27]$.

\subsection{Preparation of Ti3AIC2 MAX phase}

Ti3AIC2 MAX phase was performed by the procedure described in [25]. Powders of titanium, aluminum and graphite were mixed in molar ratio 3.00:1.10:1.88 with zirconia balls (diameter $3 \mathrm{~mm}$ ) by ball milling at $60 \mathrm{rpm}$ during 3 hours in a plastic jar. The mixed powders were putted into alumina boat and transferred to a tube furnace (4016T, Clasic CZ). Before synthesis, vacuum pumping was carried out twice to a pressure of 2 mbar, followed by a purge with argon. The MAX phase synthesis was accomplished by heating the mixture at $10^{\circ} \mathrm{C} / \mathrm{min}$ to $1550^{\circ} \mathrm{C}$ in argon atmosphere followed by 2 hours holding period at $1550^{\circ} \mathrm{C}$ and cooling down to room temperature at $10^{\circ} \mathrm{C} / \mathrm{min}$.

\section{Preparation of MXenes flakes}

Ti3C2 MXene was synthesized using the MILD method described in [26]. Before selective etching of aluminum from Ti3AIC2 MAX phase, the MAX phase ceramics were crushed by mortar and pestle and sieved to separate the particles of size below $45 \mu \mathrm{m}$. The etchant was prepared by dissolving $300 \mathrm{mg}$ of LiF $(99.98 \%$ metals basis, Alfa Aesar) in $6 \mathrm{~mL}$ of $6 \mathrm{M} \mathrm{HCl}$ in the plastic centrifuge tube. Then, the $300 \mathrm{mg}$ of sieved MAX phase powder (particle size below $45 \mu \mathrm{m}$ ) was added to prepared etchant and stirred at $22^{\circ} \mathrm{C}$ for 24 hours. After etching, the MXene were washed several times until the $\mathrm{pH}$ of the supernatant reached 5.5. Delamination of MXene flakes was carried out by hand shaking of the sample ( $15 \mathrm{~mL}$ DI water in $50 \mathrm{~mL}$ plastic test tube) for 5 min followed by $15 \mathrm{~min}$ of centrifugation at $3500 \mathrm{rpm}$ to precipitate and separate the non-delaminated MXene.

\section{Surface grafting and MXene thin film preparation}

The colloidal suspension of MXene flakes $(2 \mathrm{~mL}, 5 \mathrm{~g} / \mathrm{L})$ was mixed with a $1 \mathrm{~mL}$ of a radical source $(1 \mathrm{mM}$ solution) in a 9:1 water/methanol mixture and activated under the continuous mixing for different time intervals. After the modification, the MXene flakes were collected by centrifugation (15 min, $5000 \mathrm{rpm}$ ) and 3x subsequently washed by water and a methanol. For coating preparation, the purified MXene flakes were dispersed $(1 \mathrm{~g} / \mathrm{L})$ in water/methanol mixture $(1: 1 \mathrm{ratio})$ and sprayed on previously cleaned glass or silicon surfaces using home-made equipment.

\subsection{Measurement techniques}

For the characterization of the solutions, the UV/Vis/NIR spectroscopy technique was used in the spectral range $300-1000 \mathrm{~nm}$, scanning rate $480 \mathrm{~nm} \mathrm{~min}{ }^{-1}$, data collection interval $1 \mathrm{~nm}$. The solutions were measured in $1 \mathrm{~cm}$ quartz cells.

TEM images were obtained on a JEOL JEM-1010 transmission electron microscope with a SIS MegaView III digital camera. 
Fourier transform infrared (FTIR)-attenuated total reflection (ATR) spectra were recorded using a Nicolet 6700 spectrometer (Thermo Scientific, France) with a Smart ATR accessory device (1000 scans and $4 \mathrm{~cm}-1$ resolution).

The crystal structure of Ti3AIC2 MAX phase and Ti3C2 MXene phase were characterized by X-ray diffractometer (Empyrean, Malvern Panalytical) using $\mathrm{Cu} \mathrm{K}<\alpha>$ radiation (photon energy $-8.04 \mathrm{keV}$, wavelength $\lambda=1.54151 \AA, U=45 \mathrm{kV}, \mathrm{I}=30 \mathrm{~mA}$ ) in grazing incidence $X$-ray diffraction mode and in $2 \theta-\theta$ mode. The incident angle was 0.85 corresponding to $\sim 10 \mathrm{~mm}$ of sample irradiation.

X-ray photoelectron spectroscopy (XPS) was performed using an Omicron Nanotechnology ESCAProbeP spectrometer fitted with a monochromated Al K alpha X-ray source at $1486.7 \mathrm{eV}$. MXene flake samples were deposited on Si wafers, the analyzed area had dimensions of $2 \times 3 \mathrm{~mm} 2$.

\section{RESULTS AND DISCUSSION}

The schematic representation of main experimental concept is given in Figure 1. The MXene flakes were dispersed in an aqueous methanol solution containing radical source and then activated by external triggering. Excitation of the surface MXene increases the energy of electrons on the surface, which in turn leads to homolytic of radical source and the appearance of reactive radicals. The created radicals were then grafted to the MXene surface. Moreover, simultaneous triggering and radical grafting can result in substitution of terminated groups on flake surface, leading to pronounced modification of MXene surface composition.

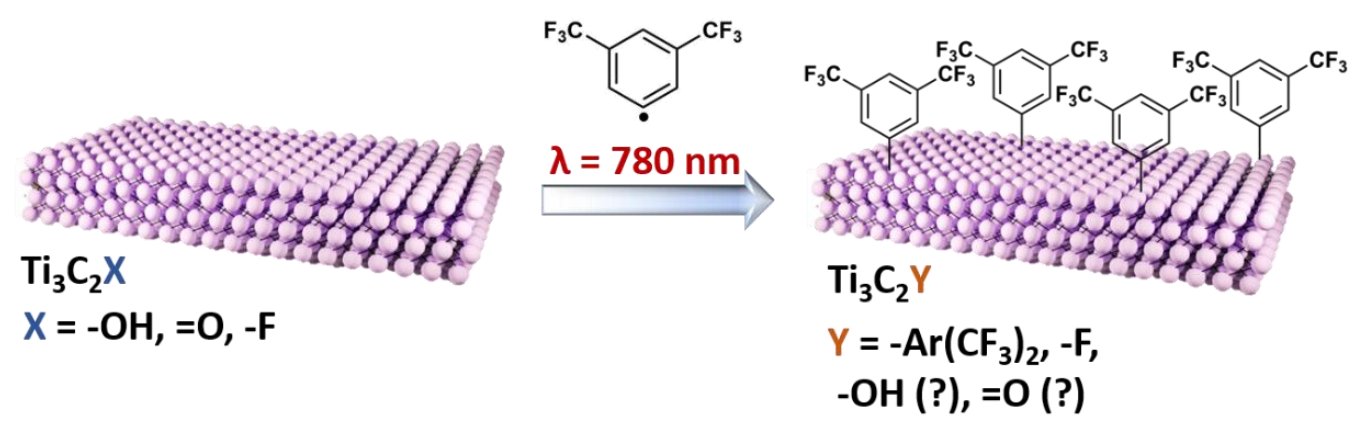

Figure 1 Schematic representation of proposed experimental concept

UV/Vis spectra of pristine MXenes in the water/methanol solution (Figure 2A - insert) were measured to determine the wavelength position of MXene absorption band. Next, the UV spectra were measured after different modification times (Figure 2A). We can observe that the absorption peak gradually shifts to the blue region with increasing modification time. After 3 hours of modification, the peak shift no longer occurred. The grafting of MXene flakes surface was further confirmed by IR measurements. IR spectra are presented in the Figure 2B. Pristine MXene contains peaks at $1740(\mathrm{C}=\mathrm{O}), 1408(\mathrm{O}-\mathrm{H})$, and $595(\mathrm{Ti}-\mathrm{O}) \mathrm{cm}^{-1}$ related to terminated oxidized groups. After radical grafting the appearance of new, well pronounced absorption bands on modified flakes indicate the presence of grafted chemical moieties.

The intensity of characteristic band, located at $1275 \mathrm{~cm}^{-1}$ (C-F stretch in $-\mathrm{CF}_{3}$ ), is plotted against the time of radicals grafting in Figure 2B insert. Given dependency corresponds well with UV/Vis results - apparent increase of band intensity during the first $30 \mathrm{~min}$ of triggering and it's saturation after 3 hours of reaction. So, results of UV/Vis and IR spectroscopy indicate that radicals grafting of MXene flakes mainly occurs at the initial stages of triggering and is further enhanced under the prolongation of triggering time up to 3 hours.

In addition, the structure and crystallinity of MXene flakes were evaluated by TEM and XRD, XPS measurements before and after radicals grafting. TEM analysis confirm the preservation of flake structure during the grafting. Results of XPS and XRD analysis are in a good agreement and approve the successful grafting of organic radicals to the surface of MXene. 

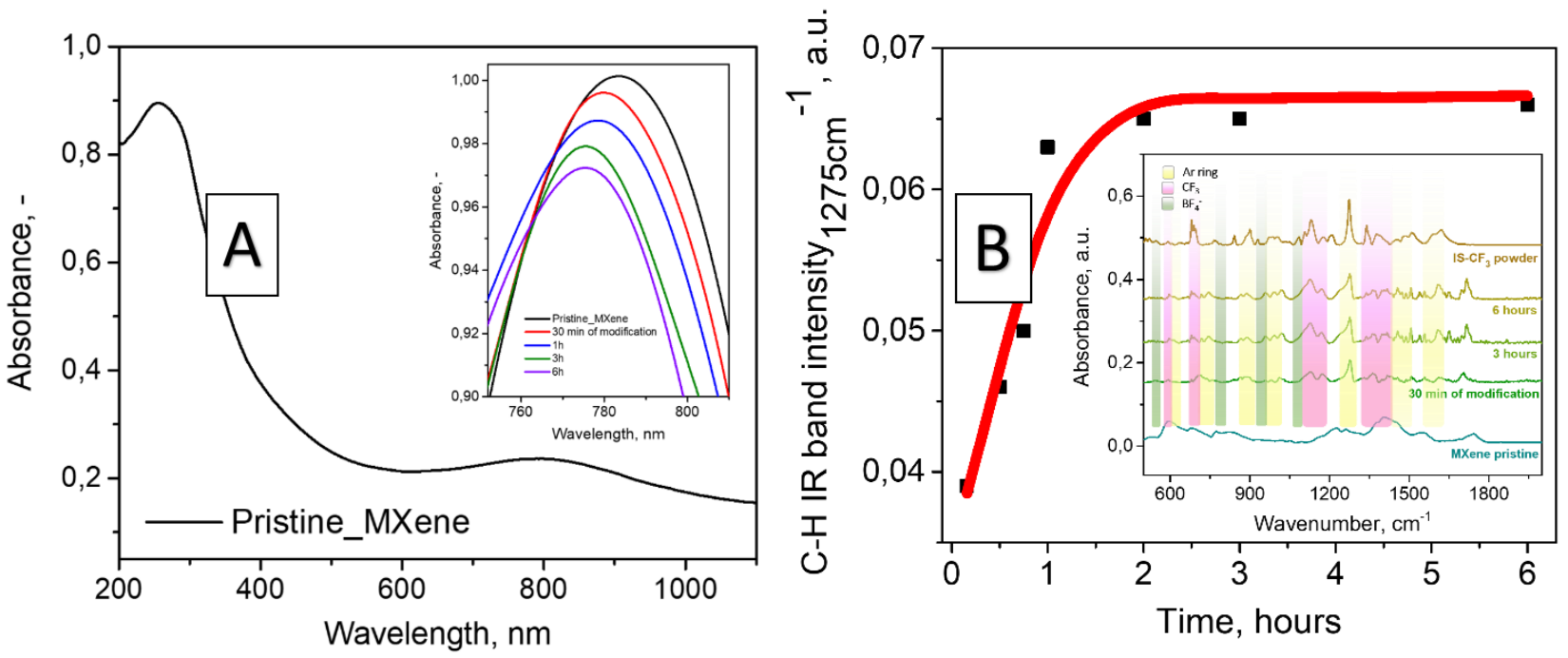

Figure 2 (A) UV/Vis absorption spectrum of MXene after different time of grafting (insert shows the full UV/Vis absorption spectrum of pristine flakes suspension); (B) IR spectra, measured on MXene flakes after the different time of radicals grafting (insert show the time dependent changes of characteristic $(1275 \mathrm{~cm}-1)$

IR band intensity)

\section{CONCLUSION}

A surface modification of MXene flakes under radical attack has been proposed. The mixture of flakes with the radical source was activated for different times. As a result of chemical bond homolysis the active radicals appear, attack the MXene surface and change the chemical composition of the surface terminated groups. Due to the high MXene catalytic potential the proposed experimental route is very simple and does not require high-energy cleaning of the flake surface. As a model example the fluor-substituted radicals were used, resulting in hydrophobization of MXene flakes surface. The proposed method provides the creation of conductive, superhydrophobic and water-repellent coatings with improved resistance of MXene to oxidation.

\section{ACKNOWLEDGEMENTS}

This work was financially supported by the Internal Grant Agency UCT № 126882010.

\section{REFERENCES}

[1] MIRO, P., AUDIFFRED, M., HEINE, T. An atlas of two-dimensional materials. Chem. Soc. Rev. 2014, vol. 43, pp. 6537-6554.

[2] SINGH, A. K., MATHEW, K., ZHUANG, H. L., HENNIG, R.G. Computational Screening of 2D Materials for Photocatalysis. J. Phys. Chem. Lett. 2015, vol. 6, pp. 1087-1098.

[3] SCHULMAN, D. S., ARNOLD, A. J., DAS, S. Contact engineering for 2D materials and devices. Chem. Soc. Rev. 2018, vol. 47, pp. 3037-3058.

[4] MAS-BALLESTE, R., GOMEZ-NAVARRO, C., GOMEZ-HERRERO, J., ZAMORA, F. 2D materials: to graphene and beyond. Nanoscale. 2011, vol. 3, pp. 20-30.

[5] CAI, Z., LIU, B., ZOU, X., CHENG, H-M. Chemical Vapor Deposition Growth and Applications of TwoDimensional Materials and Their Heterostructures. Chem. Rev. 2018, vol. 118, pp. 6091-6133.

[6] KUMAR, K. S., CHOUDHARY, N., JUNG, Y., THOMAS, J. Recent Advances in Two-Dimensional Nanomaterials for Supercapacitor Electrode Applications. CS Energy Lett. 2018, vol. 3, pp. 482-495.

[7] ZHAO, H., CHEN, X., WANG, G., QIU, Y., GUO, L. Two-dimensional amorphous nanomaterials: synthesis and applications. 2D Mater. 2019, vol. 6, p. 032002. 
[8] POMERANTSEVA, E., GOGOTSI, Y. Two-dimensional heterostructures for energy storage. Nat. Energy. 2017, vol. 2, p. 17089.

[9] WANG, H., LIU, F., FU, W., FANG, Z., ZHOU, W., LIU, Z. Two-dimensional heterostructures: fabrication, characterization, and application. Nanoscale. 2014, vol. 6, pp. 12250-12272.

[10] CHIMENE, D., ALGE, D. L., GAHARWAR, A. K. Two-Dimensional Nanomaterials for Biomedical Applications: Emerging Trends and Future Prospects. Adv. Mater. 2015, vol. 27, pp. 7261-7284.

[11] GERSTNER E. Nobel Prize 2010: Andre Geim \& Konstantin Novoselov. Nature Physics. 2010, vol. 6, p. 836.

[12] SUN, Z., CHANG, H. Graphene and Graphene-like Two-Dimensional Materials in Photodetection: Mechanisms and Methodology. ACS Nano. 2014, vol. 8, pp. 4133-4156.

[13] GRIGORENKO, A. N., POLINI, M., NOVOSELOV, K. S. Graphene plasmonics. Nat. Photonics. 2012, vol. 6, pp. 749-758.

[14] PAPAGEORGIOU, D. G., KINLOCH, I. A., YOUNG, R. J. Mechanical properties of graphene and graphenebased nanocomposites. Prog. Mater. Sci. 2017, vol. 90, pp. 75-127.

[15] CUI, S., MAO, S., JU, G., CHEN, J. Graphene Coupled with Nanocrystals: Opportunities and Challenges for Energy and Sensing Applications. J. Phys. Chem. Lett. 2013, vol. 4, pp. 2441-2454.

[16] LEE, S-M., KIM, J-H., AHN, J-H. Graphene as a flexible electronic material: mechanical limitations by defect formation and efforts to overcome. Mater. Today. 2015, vol. 18, pp. 336-344.

[17] MOUCHEL, P., KEMEL, M., SEMAAN, G., SALHI, M., FLOHIC, M. L., SANCHEZ, F. Limitations of graphene nanocoated optical tapers for high-power nonlinear applications. Opt. Mater. X. 2019, vol. 1, p. 100003.

[18] NAGUIB, M., KURTOGLU, M., PRESSER, V., LU, J., NIU, J., HEON, M., HULTMAN, L., GOGOTSI, Y., BARSOUM, M. W. Two-Dimensional Nanocrystals: Two-Dimensional Nanocrystals Produced by Exfoliation of Ti3AIC2. Adv. Mater. 2011, vol. 23, pp. 4248-4253.

[19] NAGUIB, M., COME, J., DYATKIN, B., PRESSER, V., TABERNA, P-L., SIMON, P., BARSOUM, M. W., GOGOTSI, Y. MXene: a promising transition metal carbide anode for lithium-ion batteries. Electrochem. commun. 2012, vol. 16, pp. 61-64.

[20] CHERTOPALOV, S., MOCHALIN, V. N. Environment-Sensitive Photoresponse of Spontaneously Partially Oxidized Ti3C2 MXene Thin Films. ACS Nano. 2018, vol. 12, pp. 6109-6116.

[21] LIU, J., ZHANG, H-B., SUN, R., LIU, Y., LIU, Z., ZHOU, A., YU, Z-Z. Hydrophobic, Flexible, and Lightweight MXene Foams for High-Performance Electromagnetic-Interference Shielding. Adv. Mater. 2017, vol. 29, p. 1702367.

[22] LIU, R., MIAO, M., LI, Y., ZHANG, J., CAO, S., FENG, X. Ultrathin Biomimetic Polymeric Ti3C2Tx MXene Composite Films for Electromagnetic Interference Shielding. ACS Appl. Mater. Interfaces. 2018, vol. 10, pp. 44787-44795.

[23] XIONG, D., LI, X., BAI, Z., LU, S. Recent Advances in Layered Ti3C2Tx MXene for Electrochemical Energy Storage. Small. 2018, vol. 14, p. 1703419.

[24] GUSELNIKOVA, O., TRELIN, A., MILIUTINA, E., ELASHNIKOV, R., SAJDL, P., POSTNIKOV, P., KOLSKA, Z., SVORCIK, V., LYUTAKOV, O. Plasmon-Induced Water Splitting一through Flexible Hybrid 2D Architecture up to Hydrogen from Seawater under NIR Light. ACS Appl. Mater. Interfaces. 2020, vol. 12, pp. 28110-28119.

[25] ALHABEB, M., MALESKI, K., ANASORI, B., LELYUKH, P., CLARK, L., SIN, S., GOGOTSKI, Y. Guidelines for Synthesis and Processing of Two-Dimensional Titanium Carbide (Ti3C2Tx MXene). Chem. Mater. 2017, vol. 29, pp. 7633-7644.

[26] DONG, Y., CHERTOPALOV, S., MALESKI, K., ANASORI, B., HU, L., BHATTACHARYA, S., RAO, A. M., GOGOTSKI, Y., MOCHALIN, V. N., PODILA, R. Saturable absorption in 2D Ti3C2 MXene thin films for passive photonic diodes. Adv. Mater. 2018, vol. 30, p. 1705714.

[27] OlShtREM, A., GUSElNIKOVA, O., POSTNIKOV, P., TRELIN, A., YUSUBOV, M., KALACHYOVA, E., LAPCAK, L., CIESLAR, M., ULBRICH, P., SVORCIK, V., LYUTAKOV, A. Plasmon-assisted grafting of anisotropic nanoparticles - spatially selective surface modification and the creation of amphiphilic SERS nanoprobes. Nanoscale. 2020, vol. 12, pp. 14581-14588. 